Relations industrielles

Industrial Relations

\title{
La face cachée de l'organisation : groupes, cliques et clans, par Luc BRUNET et André SAVOIE, Montréal : Les Presses de l’Université de Montréal, 2003, 158 p., ISBN 2-7606-1866-8.
}

\section{Richard Pépin}

Volume 60, numéro 3, été 2005

URI : https://id.erudit.org/iderudit/012161ar

DOI : https://doi.org/10.7202/012161ar

Aller au sommaire du numéro

Éditeur(s)

Département des relations industrielles de l'Université Laval

ISSN

0034-379X (imprimé)

1703-8138 (numérique)

Découvrir la revue

Citer ce compte rendu

Pépin, R. (2005). Compte rendu de [La face cachée de l'organisation :

groupes, cliques et clans, par Luc BRUNET et André SAVOIE, Montréal : Les

Presses de l'Université de Montréal, 2003, 158 p., ISBN 2-7606-1866-8.] Relations

industrielles / Industrial Relations, 60(3), 574-576.

https://doi.org/10.7202/012161ar

Tous droits réservés (C) Département des relations industrielles, Université Laval, 2005
Ce document est protégé par la loi sur le droit d'auteur. L'utilisation des services d’Érudit (y compris la reproduction) est assujettie à sa politique d'utilisation que vous pouvez consulter en ligne.

https://apropos.erudit.org/fr/usagers/politique-dutilisation/ 
La face cachée de l'organisation : groupes, cliques et clans, par Luc BRUNET et André SAVOIE, Montréal : Les Presses de l'Université de Montréal, 2003, 158 p., ISBN 2-7606-1866-8.

Même si au cours de leur carrière la plupart des gens a déjà fait partie au moins d'un groupe informel en milieu de travail, c'est-à-dire d'un groupe qui émerge sans avoir été planifié par l'autorité formelle, on ne connaît encore que très peu de choses sur l'émergence, le développement et les effets des groupes informels dans les organisations. C'est précisément ce vide que cherche à combler ce volume. En s'appuyant sur près de dix années d'études sur le terrain menées par une équipe de recherche de l'Université de Montréal sur le développement naturel des groupes en milieu de travail, cet ouvrage présente une synthèse fort bien appuyée des connaissances empiriques acquises depuis les années 1930 sur un phénomène social qui, même s'il fait partie intégrante de la vie organisationnelle, demeure néanmoins encore très peu étudié.

À cette fin, l'ouvrage, qui comporte cinq chapitres, situe tout d'abord la notion de groupe informel à l'intérieur du développement de la psychologie du travail et des théories de l'organisation. Par la suite, il définit la nature des groupes non officiels dans les organisations, puis attire l'attention tour à tour sur l'émergence et l'influence de la culture du groupe informel, sur les fonctions, les rôles et les effets des groupes informels sur le fonctionnement de l'organisation, et enfin sur le rôle du groupe informel dans l'accès, la rétention et la déformation possible de l'information en milieu organisationnel.

Le premier chapitre traite de la réalité informelle des organisations. On y apporte des distinctions utiles entre la structure formelle et la structure informelle en rappelant qu'à toute structure formelle (ou ensemble de relations prescrites et officialisées entre les unités ou les membres d'une organisation) se greffe inévitablement une structure informelle (ou liens non mandatés en vertu des règles de l'organisation formelle et qui émergent spontanément de manière à satisfaire les besoins des individus). À ce propos, les auteurs rappellent à juste titre que les études réalisées à l'usine Western Electric de Hawthorne sous la gouverne d'Elton Mayo (1933), et plus tard sous celle de Roethlisberger et Dickson (1939), ainsi que l'étude empirique réalisée par l'Institut Tavistock dans les mines de charbon britanniques vers la fin des années 1940, furent les premières à mettre en évidence l'inévitabilité des relations informelles et leur impact sur l'efficacité de l'organisation.

Le deuxième chapitre se concentre sur la définition et la nature du groupe informel. Les auteurs associent d'entrée de jeu les groupes informels aux groupes restreints, c'est-à-dire à des structures sociales au sein desquelles les êtres humains se retrouvent afin de répondre à certains besoins de la vie en milieu organisationnel. À ce titre, le groupe informel comporte trois caractéristiques : 1) la présence d'interactions psychologiques directes entre les membres (ce qui implique un nombre relativement restreint de personnes); 2) l'interdépendance des membres par rapport à une cible commune (qu'il s'agisse par exemple de s'épauler et de se soutenir ou de s'unir pour accomplir une tâche); et 3) un champ psychologique en constante évolution (sous l'influence de mécanismes normatifs propres au groupe). Les auteurs soulignent qu'en comblant des besoins relationnels (à savoir les besoins de similitude, de complémentarité et de protection) laissés pour compte par l'organisation, le groupe informel constitue un dispositif de gestion des intérêts individuels au travail. Ils précisent que la non-satisfaction de ces besoins ainsi qu'un environnement de travail 
caractérisé, entre autres, par un degré élevé et rigide de formalisation, une distribution restreinte des ressources, la proximité physique des travailleurs et le caractère plus ou moins stressant du travail peuvent affecter l'émergence et la nature des groupes informels. Les auteurs précisent à ce propos qu'il existe plusieurs types de groupes informels en milieu de travail et que ces derniers peuvent prendre des noms variés selon les objectifs poursuivis. On retrouve ainsi des cliques, des claques, des cabales, des clans, des fonctionnaires, des loyalistes et des factions. Ces différents groupes peuvent se liguer entre eux pour former des coalitions en vue de combattre les incertitudes qui les frappent.

La culture du groupe informel fait l'objet du chapitre 3. Les auteurs soutiennent ici que la culture des groupes informels agit de façon identique à la culture organisationnelle dans laquelle ceux-ci se trouvent. Ainsi, les rites, les rituels, les traditions et le partage des symboles en représentent des caractéristiques importantes. L'influence de ces éléments culturels permettrait à ces groupes d'établir un contrôle social plus ou moins conscient qui incite les membres à se conformer aux attentes à leur endroit. Les données empiriques indiquent ici que la culture propre au groupe informel semble davantage encourager les membres à être eux-mêmes et à innover qu'à se plier aux impératifs de 1'organisation; qu'elle génère des attentes de réalisation de soi significativement plus fortes que celle des autres groupes et qu'elle ne véhicule pas plus d'attentes d'acquisition de pouvoir que celle en vigueur dans les groupes formels.

Le chapitre 4 se concentre sur les apports du groupe informel. Les études indiquent que le groupe informel peut être vu comme un dispositif naturel de soutien social, instrumental, politique et de prévention des conflits pour ses membres. En ce sens, il permet aux membres de se sentir appuyés et de développer des amitiés, c'est-à-dire des relations empreintes d'intimité, de plaisir, de respect et de franchise au travail. L'appartenance au groupe informel permet ainsi aux travailleurs de se percevoir comme étant plus en contrôle de leur environnement, c'est-à-dire d'être plus en mesure d'interpréter la réalité organisationnelle. Pour ces raisons, le groupe informel semble favoriser le bien-être, l'adaptation de ses membres et leur évite de vivre l'aliénation au travail. Les auteurs soulignent que les membres des groupes informels se disent plus satisfaits de leur travail que les non-membres; développent des normes qui ne s'opposent pas, de prime abord, au bon fonctionnement de leur entreprise ; ne se voient pas, a priori, comme une source de contre-pouvoir face à leur organisation; et ne s'opposent pas d'emblée aux changements organisationnels.

Le cinquième et dernier chapitre traite de la gestion de l'information par les groupes informels. À ce propos, les auteurs rapportent notamment que les membres de groupes informels de bas niveau hiérarchique ont accès à plus d'information privilégiée, c'est-à-dire à de l'information à caractère organisationnelle n'étant pas accessible à tous les travailleurs; les travailleurs qui effectuent le plus de rétention d'information sont les membres de groupes informels faisant partie d'un réseau informel; la rétention d'information dans le groupe informel pourrait être associée à des climats de travail caractérisés par la méfiance; les membres de groupes informels ne déforment pas plus l'information que les autres membres de l'organisation.

Dans la conclusion de l'ouvrage, les auteurs rappellent essentiellement trois points. Premièrement, le groupe informel répond avant tout à des besoins sociaux auxquels la structure formelle de l'organisation ne peut répondre. Deuxièmement, le groupe informel 
résiste à la structure formelle lorsque des failles organisationnelles importantes, comme un mauvais climat de travail ou une structure d'autorité trop rigide, se développent et provoquent des confrontations. Troisièmement, puisqu'il agit comme un dispositif naturel de soutien social au travail permettant aux membres de mieux interpréter leur environnement, de vivre moins de stress et de mieux orienter leur comportement, le groupe informel doit être considéré comme un allié dans les changements qui affectent l'organisation.

Dans l'ensemble, La face cachée de l'organisation est à mon sens un excellent livre. Il convient ici de féliciter les auteurs ainsi que les membres de leur équipe de recherche pour la rigueur et la minutie qu'ils ont déployées au fil des années pour commencer à lever le voile sur ce phénomène social à l'évidence commun et significatif, mais curieusement encore méconnu, de l'informel dans les organisations. La démarche de recherche qui appuie cet ouvrage de synthèse ne laisse aucun doute sur la fiabilité des résultats. Dans un langage accessible à un large auditoire, cet ouvrage contribue à démystifier la dimension informelle des organisations : il en circonscrit la nature, certaines composantes et caractéristiques clés, quelques facteurs d'émergence et plusieurs effets dans l'organisation. Le livre a aussi les défauts de ses qualités. S'il est petit, relativement facile à lire et fort descriptif, on n'y retrouve toutefois pas beaucoup de conseils susceptibles d'aider les gestionnaires à mieux prendre en compte cette dimension informelle de l'organisation. Par exemple, j'aurais aimé y retrouver quelques suggestions sur les conditions à créer dans les organisations pour inciter les groupes informels à s'inscrire dans une logique de collaboration avec la structure formelle. Il faut reconnaître toutefois qu'étant donné l'état jusque-là embryonnaire des connaissances sur la dimension informelle des organisations, ce petit livre joue fort bien son rôle : il éclaire avec force et détails notre compréhension de cette face cachée de l'organisation.

RICHARD PÉPIN

Université du Québec à Trois-Rivières

\section{Initiative individuelle et formation,} sous la direction de Fabienne BERTON, Mario CoRREIA, Corinne LeSPESSAILles et Madeleine MAILlebouis, Paris : L'Harmattan, 2004, 289 p., ISBN 2-74757275-7.

Utile et salutaire sont les deux premiers mots qui viennent à l'esprit pour caractériser cet ouvrage. Utile car il fournit un matériau riche et diversifié, appuyé par d'amples bibliographies thématiques commentées, permettant de faire le point des connaissances et débats sur l'individualisation dans le champ de la formation. Le chercheur, l'enseignant, l'étudiant mais aussi le professionnel peuvent y puiser, chacun selon son centre d'intérêt. Salutaire car il permet de se repérer dans les controverses scientifiques et sociales autour de notions fortement débattues mais aussi de catégories souvent encore floues.
Comme d'autres ouvrages issus du Conservatoire national des arts et métiers - CNAM - (sur les formations en alternance, les métiers de la formation, l'orientation professionnelle), celui-ci est le produit d'une rencontre entre un centre de documentation, proposant sur une thématique précise une bibliographie structurée, et un collectif de chercheurs venant éclairer les différentes facettes du problème.

S'attaquer aujourd'hui à la question de l'initiative individuelle est particulièrement bienvenu. D'une part parce que cette notion a été souvent liée, si ce n'est 